\title{
Evaluation of the cross-sectional area of acromion process for shoulder impingement syndrome
}

\author{
Young $\mathrm{JOo}^{1, *}$, Hyung Rae $\mathrm{Cho}^{2, *}$, and Young Uk Kim ${ }^{1}$ \\ 'Department of Anesthesiology and Pain Medicine, Catholic Kwandong University International St. Mary's Hospital, Catholic Kwandong University \\ College of Medicine, Incheon, Korea \\ ${ }^{2}$ Department of Anesthesiology and Pain Medicine, Myongji Hospital, Hanyang University College of Medicine, Goyang, Korea
}

Received July 9, 2019

Revised September 10, 2019

Accepted September 16, 2019

\section{Correspondence}

Young Uk Kim

Department of Anesthesiology and Pain Medicine, Catholic Kwandong University International St. Mary's Hospital, Catholic Kwandong University College of Medicine, 25 Simgok-ro 100beon-gil, Seo-gu, Incheon 22711, Korea

Tel: +82-32-290-3570

Fax: +82-32-290-3568

E-mail: uk201@hanmail.net

*These authors contributed equally to this work.
Background: Anatomic changes in the acromion have been considered a main cause of shoulder impingement syndrome (SIS). To evaluate the relationship between SIS and the acromion process, we devised a new morphological parameter called the acromion process cross-sectional area (APA). We hypothesized that the APA could be an important morphologic diagnostic parameter in SIS.

Methods: We collected APA data from 95 patients with SIS and 126 control subjects who underwent shoulder magnetic resonance imaging (MRI). Then we measured the maximal cross-sectional area of the bone margin of the acromion process on MRI scans.

Results: The mean of APAs were $136.50 \pm 21.75 \mathrm{~mm}^{2}$ in the male control group and $202.91 \pm 31.78 \mathrm{~mm}^{2}$ in the male SIS group; SIS patients had significantly greater APAs $(P<0.001)$. The average of APAs were $105.38 \pm 19.07 \mathrm{~mm}^{2}$ in the female control group and $147.62 \pm 22.90 \mathrm{~mm}^{2}$ in the female SIS group, and the SIS patients had significantly greater APAs $(P<0.001)$. The optimal APA cut-off in the male group was $165.14 \mathrm{~mm}^{2}$ with $90.2 \%$ sensitivity, $91.4 \%$ specificity, and an area under the curve (AUC) of 0.968. In the female group, the optimal cut-off was $122.50 \mathrm{~mm}^{2}$ with $85.2 \%$ sensitivity, $84.9 \%$ specificity, and an AUC of 0.928 .

Conclusions: The newly devised APA is a sensitive parameter for assessing SIS; greater APA is associated with a higher possibility of SIS. We think that this result will be helpful for the diagnosis of SIS.

Key Words: Acromion; Anatomy, Cross-Sectional; Diagnosis; Osteophyte; Parameter; Process; Shoulder; Shoulder Impingement Syndrome

\section{INTRODUCTION}

The acromion is a clinically important anatomical feature of the coracoacromical arch. Anatomic changes to the acromion and the acromioclavicular joint can reduce the volume of the supraspinatous opening and increase mechanical degeneration and wear of underlying soft tissue [1]. Shoulder impingement syndrome (SIS) occurs be- tween the rotator cuff and the acromion and involves the acromioclavicular joint and the coracoacromial ligament [1-4]. SIS typically leads to weakness and/or pain around the shoulder region, loss of movement in the affected shoulder, and difficulty in sleeping $[5,6]$. Osteophytes, hypertrophic changes, and bony spurs in the acromion have been considered major causes of SIS. Subacromial or acromioclavicular spurs were reported to be observed (c) This is an open-access article distributed under the terms of the Creative Commons Attribution Non-Commercial License (http://creativecommons.org/licenses/by-nc/4.0/), which permits unrestricted non-commercial use, distribution, and reproduction in any medium, provided the original work is properly cited.

(c) The Korean Pain Society, 2020
Author contributions: Young Joo: Formal analysis; Hyung Rae Cho: Methodology; Young Uk Kim: Study conception. 
in about $50 \%$ of SIS cases [6]. Most anatomic studies have focused on morphologic classifications of the acromion, such as being of a flat, curved, hooked, or convex type [7-9]. However, morphologic classification does not reflect hypertrophic changes in the acromion, such as osteophytes and bony spurs. Moreover, the shape of the acromion is not always regular, and the directions of the axis of the acromion surface cannot be determined [10]. Thus, for evaluating hypertrophic changes in the whole acromion, a new morphological parameter called the acromion process cross-sectional area (APA) was devised by measuring the maximum cross-sectional area of the acromion process. Unlike conventional morphological classifications of the acromion, APA reflects hypertrophic changes. We hypothesized that the APA would be a key morphologic parameter in the diagnosis of SIS. There are no previous reports of an association between SIS and the whole crosssectional acromion area as a morphologic parameter on magnetic resonance imaging (MRI). Thus, we compared the APA between SIS patients and healthy controls using MRI. The aims of this retrospective study were to investigate the optimal cut-off value of APA in patients with SIS and to evaluate the usefulness of the APA as an objective diagnostic tool in determining SIS.

\section{MATERIALS AND METHODS}

\section{Patients}

We registered this study at the Catholic Kwandong University College of Medicine, Republic of Korea (IS17RISI0042), and the University's Institutional Review Board reviewed and approved the research protocol. We retrospectively reviewed patients who had visited Catholic Kwandong University International St. Mary's Hospital from March 2014 to June 2017 and who had been diagnosed with SIS. All patients were enrolled after the diagnosis of SIS was confirmed by two experienced, board-certified, musculoskeletal radiologists. The SIS group comprised 95 patients (41 males and 54 females).

The inclusion criteria for the SIS group were: 1) posi- tive shoulder impingement signs (Hawkins-Kennedy test or Neer's test); 2) shoulder pain during active arm elevation; 3) a shoulder MRI taken within 12 months of the first diagnosis and available for review; 4) a painful arc; and 5) weakness or pain with resisting scapular plane abduction on internal humeral rotation or resisting isometric external rotation. Exclusion criteria were: 1) a history of upper arm fracture; 2) shoulder pain with passive or active cervical spine motion; 3 ) a history of shoulder surgery; 4) evidence of frozen shoulder; and 5) a full-thickness rotator cuff tear.

To compare the APAs between patients with and without SIS, we also enrolled a control group of individuals who had undergone shoulder MRI as a part of routine medical examinations. We only enrolled patients in the control group who had no complaints of upper arm or shoulder pain, without matching them to the SIS group. The control group comprised 126 individuals (70 males and 56 females) with mean ages of $53.2 \pm 8.7$ years (range, 33-73 yr) in the male group and $53.3 \pm 7.0$ years (range, $39-71 \mathrm{yr}$ ) in the female group (Table 1). We excluded patients from the control group if they reported any known shoulder pathology or met any of the above mentioned exclusion criteria.

\section{MRI scanning protocol}

All MRI scans were taken at the Department of Orthopedics and Shoulder Center, Catholic Kwandong University International St. Mary's Hospital. In both groups, the MRIs were taken with the same technique and position using proton density fast spin-echo (FSE) imaging. The MRI scans were taken on 3T scanners (Magnetom Skyra, Sonata, Biograph, Avanto [Siemens Healthcare GmbH, Erlangen, Germany]; Philips Ingenia [R4] [Philips Medical Systems, Best, The Netherlands]) with a dedicated shoulder coil (Med Rad Multipurpose Array, Indianola, PA). Pulse sequence parameters included oblique coronal FSE fat suppression (repetition time [TR]: 3,000 msec, echo time [TE]: 90 msec, echo train length [ETL]: 15, matrix: 448 $\times 270$, slice thickness: $4 \mathrm{~mm}$, field of view: $16 \mathrm{~cm}$ ). The following other parameters were used: a $0.4 \mathrm{~mm}$ intersection gap, $2,700 \mathrm{msec} / 95 \mathrm{msec} \mathrm{TR} / \mathrm{TE}, 300 \mathrm{~cm} \times 300 \mathrm{~cm}$ field of

Table 1. Comparison of the Characteristics of Control, and SIS Group

\begin{tabular}{|c|c|c|c|c|}
\hline \multirow{2}{*}{ Parameter } & \multicolumn{2}{|c|}{ Male } & \multicolumn{2}{|c|}{ Female } \\
\hline & Control group $(n=70)$ & SIS group $(n=41)$ & Control group $(n=56)$ & SIS group $(n=54)$ \\
\hline Age (yr) & $53.2 \pm 8.7$ & $57.6 \pm 6.9$ & $53.3 \pm 7.0$ & $58.1 \pm 7.4$ \\
\hline $\operatorname{APA}\left(\mathrm{mm}^{2}\right)$ & $136.5 \pm 21.8$ & $202.9 \pm 31.8 *$ & $105.4 \pm 19.1$ & $147.6 \pm 22.9 *$ \\
\hline
\end{tabular}

Values are presented as mean \pm standard deviation.

SIS: shoulder impingement syndrome, APA: acromion process cross-sectional area.

$\star P<0.001$. 

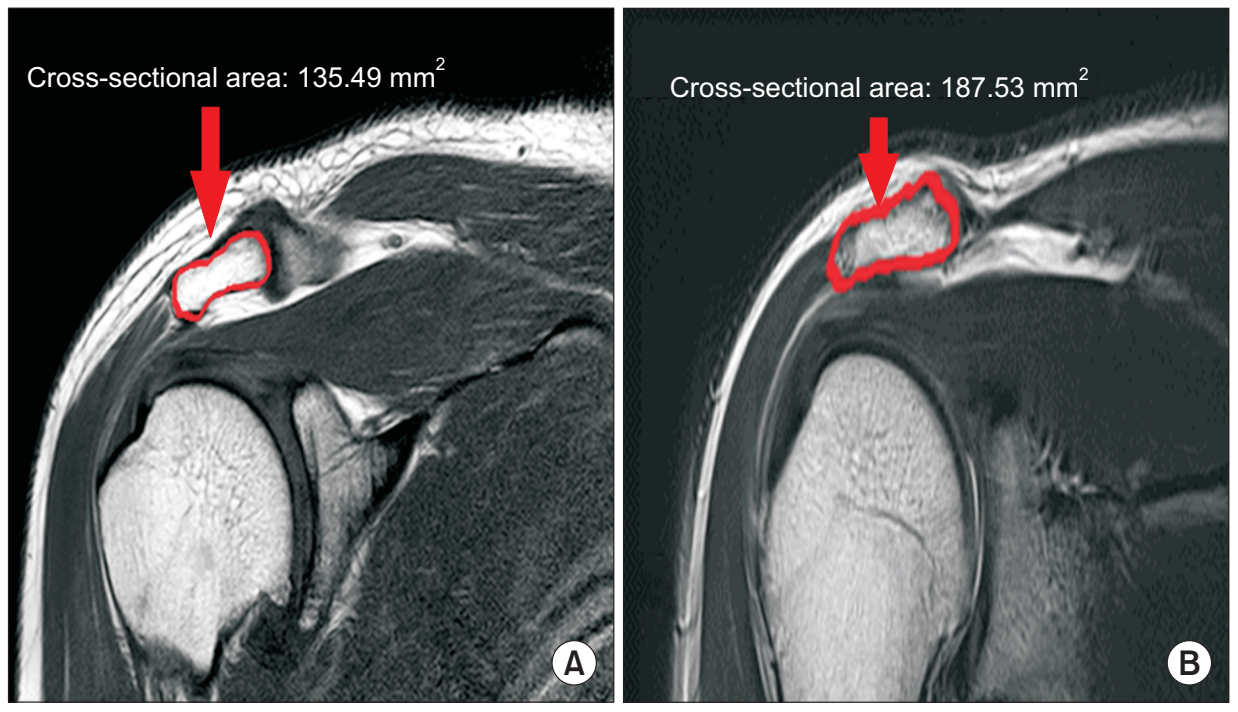

Fig. 1. Oblique coronal proton density fast spin-echo magnetic resonance imaging in acromion sections: (A) normal control male; (B) shoulder impingement syndrome.

Table 2. Sensitivity and Specificity of Each Cut-off Value of the APA in Male

\begin{tabular}{ccc}
\hline APA $\left(\mathrm{mm}^{2}\right)$ & Sensitivity $(\%)$ & Specificity $(\%)$ \\
\hline 124.95 & 100.0 & 28.6 \\
150.44 & 97.6 & 77.1 \\
156.62 & 95.1 & 81.4 \\
$165.14^{\mathrm{a}}$ & 90.2 & 91.4 \\
172.53 & 80.5 & 92.9 \\
191.67 & 61.0 & 100.0 \\
\hline
\end{tabular}

APA: acromion process cross-sectional area.

${ }^{\text {a}}$ The best cut-off point on the receiver operating characteristic curve.

view, $358 \times 512$ matrix, and 15 ETL.

\section{Image analysis}

We measured the APA in the oblique coronal proton density FSE view using a picture archiving and communications system (INFINITT Healthcare, Seoul, Korea) by outlining the acromion process (Fig. 1). Among several slices on the oblique coronal view, the one showing the maximal APA was selected and used for analysis.

\section{Statistical analyses}

According to the previous study [11], the effect size was 0.57 , and it was calculated that 18 patients per group were required to obtain a power of 0.95 , considering an alpha error of 0.05 . When the dropout rate was set at approximately $20 \%$, at least 95 medical records were reviewed for each group. Data are expressed as mean \pm standard deviation. We compared the APAs between the control and SIS groups by demographic characteristics using unpaired $t$ tests. We estimated the validity of the APA for diagnosis of
Table 3. Sensitivity and Specificity of Each Cut-off Value of the APA in Female

\begin{tabular}{ccc}
\hline APA $\left(\mathrm{mm}^{2}\right)$ & Sensitivity $(\%)$ & Specificity (\%) \\
\hline 104.85 & 100.0 & 50 \\
120.04 & 90.7 & 76.8 \\
121.81 & 87.0 & 82.1 \\
$122.50^{\mathrm{a}}$ & 85.2 & 84.9 \\
130.73 & 74.1 & 89.3 \\
156.51 & 31.5 & 100.0 \\
\hline
\end{tabular}

APA: acromion process cross-sectional area.

${ }^{a}$ The best cut-off point on the receiver operating characteristic curve.

SIS with receiver operator characteristic (ROC) curves, an optimal cut-off score, area under the curve (AUC), sensitivity, and specificity with $95 \%$ confidence intervals (CIs). We used IBM SPSS ver. 21 (IBM Corp., Armonk, NY) for the statistical analyses, and we considered $P<0.05$ to be statistically significant.

\section{RESULTS}

The mean age of the male control and SIS group was $53.2 \pm$ 8.7 and $57.6 \pm 6.9$ years, respectively. The mean age of the female control subjects was $53.3 \pm 7.0$ years and that of the female SIS patients was $58.1 \pm 7.4$ years. Sex and age were not significantly different between the groups. The average APA was $136.50 \pm 21.75 \mathrm{~mm}^{2}$ in the male control group and $202.91 \pm 31.78 \mathrm{~mm}^{2}$ in the male SIS group; the SIS patients had significantly greater APAs $(P<0.001)$ than the male controls. The average APA in the female control and SIS group was $105.38 \pm 19.07$ and $147.62 \pm 22.90 \mathrm{~mm}^{2}$, respectively; these SIS patients also had significantly greater APAs $(P<0.001)$ than the female controls (Table 1$)$. 
Regarding the validity of the APA as a predictor of SIS, ROC curve analysis showed that the optimal cut-off value of the APA in males was $165.14 \mathrm{~mm}^{2}$ with $90.2 \%$ sensitivity, $91.4 \%$ specificity (Table 2), and an AUC of 0.968 (95\% CI: 0.9410.995), and in females, it was $122.50 \mathrm{~mm}^{2}$ with $85.2 \%$ sensitivity, $84.9 \%$ specificity (Table 3 ), and an AUC of 0.928 ( $95 \%$ CI: 0.882-0.974).

\section{DISCUSSION}

There are no objective morphologic parameters to indicate acromion process hypertrophic changes. We believed that the cross-sectional area of the acromion process could be an objective, precise, clear measurement parameter for evaluating hypertrophic changes in the acromion process. In our present research, we measured the APA from oblique coronal proton density FSE images. To our best knowledge, an association between SIS and APA as a morphologic parameter on MRI has not been reported previously.

SIS is a common disorder in elderly and middle-aged populations that usually involves shoulder pain $[10,12]$. Any abnormality that disturbs the relationship of the coracoacromial arch may lead to SIS [9]. The acromion process projects forward at right angles from the lateral end of the scapular spine. It is an important component of the subacromial space, which forms the superior boundary of the coracoacromial arch [9]. The coracoacromial ligament spans between the coracoid process and the tip of the acromion to form the coracoacromial arch $[13,14]$. The space below the arch gives passage to the rotator cuff tendons of muscles [9]. Morphological changes and variations in the acromion process and its relationship with the coracoid process and glenoid cavity are the most relevant determinants of the coracoacromial arch space [15]. Thus, previous studies focused on the cause-and-effect relationship between acromial morphology and SIS. Saha and Vasudeva [9] reported three types of acromial morphology, flat, curved, and hooked, and Gagey et al. [16] described a fourth type, convex. Kitay et al. [17] described the acromial tilt. Banas et al. [18] observed the frontal plane slope of the acromion process on MRI and found smaller lateral acromial angles in patients with SIS. Nyffeler et al. [19] demonstrated that the acromion process in patients with SIS appeared to have more lateral extension than that in normal controls, and they described the acromion index. Despite these numerous previous studies, they did not reflect degenerative hypertrophic changes to the acromion process, such as enthesophytes, bony spurs, and osteophytes [8].

Few studies have investigated the anatomical basis of the acromion process. Hughes et al. [5] asserted that the lack of anatomical validity to support the use of clinical evaluations may explain their poor diagnostic accuracy, and Mayerhoefer et al. [20] contended that acromial morphology is not a good descriptor of coracoaromial arch narrowing. Aydin et al. [2] found that acromion type was not an important predictor of SIS.

Our data demonstrate an association between APA and SIS; SIS patients had significantly greater APAs than those in the control subjects. Our interpretation of this association is that hypertrophy of the acromion process might be related to continuous stress, which might increase the APA. The process of acromion hypertrophy begins with mechanical stress during shoulder movements, which leads to increased force on the acromion process and extensive abrasion. This etiology could alter the morphologic features of the acromion process. Takase and Yamamoto [21] concluded that the advanced degenerative hypertrophic changes on the undersurface of the acromion process result in full-thickness cuff tears. ROC analysis showed that the APA had high diagnostic accuracy for detecting SIS (AUC: 0.968 in males and 0.928 in females). We suggest that the APA is a precise, objective, and clear morphological predictor of SIS.

The present study had several limitations. First, there might have been errors associated with measuring the APA on MRI. We attempted to measure the maximal APA at the level of the acromioclavicular joint. However, the method of analyzing a single MRI slice to measure the maximum cross-sectional area among several slices may be inconsistent due to technical problems, rater reliability, or individual anatomic variations. To overcome these shortcomings, it is necessary to measure the three dimensional (3D) reconstructed volume with less effect on cutting angle or anatomic variations. But we didn't. Since the volume in 3D can reflect hypertrophy more accurately than the maximal APA, a study using 3D reconstructed volume may be needed in the future study. Also, it is required in further studies to establish reproducibility by measuring reliability to prevent intra-rater or inter-rater error. Second, SIS represents a combination of complex pathogenic causes, including the coracoacromial ligament, subacromial space [22], and supraspinatous tendon thickness [23], but we focused only on the acromion process. Future studies should assess the association between extrinsic direct mechanical compression and intrinsic changes in the supraspinatus tendon. Third, there are different stages of SIS, but in this study, we did not compare patients with intact rotator cuffs and patients with rotator cuff tears. Forth, this study is designed to evaluate only the relevance of APA and SIS. Further prospective study about the severity of disease or pain intensity related to APA is thought to be of great help to clinicians. In spite of these limitations, this is the first research 
to document that the APA is associated with SIS.

In conclusion, APA is a new, sensitive morphological parameter for evaluating SIS. The optimal APA cut-off points were $165.14 \mathrm{~mm}^{2}$ for males ( $90.2 \%$ sensitivity, $91.4 \%$ specificity, and an AUC of 0.968 ) and $122.50 \mathrm{~mm}^{2}$ for females (85.2\% sensitivity, 84.9\% specificity, and an AUC of 0.928). We hope that this new measurement technique will be helpful for assessing SIS.

\section{CONFLICT OF INTEREST}

No potential conflict of interest relevant to this article was reported.

\section{FUNDING}

No funding to declare.

\section{ORCID}

Young Joo, https://orcid.org/0000-0001-5104-9486

Hyung Rae Cho, https://orcid.org/0000-0003-1634-7482

Young Uk Kim, https://orcid.org/0000-0003-4977-5272

\section{REFERENCES}

1. Roidis NT, Motamed S, Vaishnav S, Ebramzadeh E, Karachalios TS, Itamura JM. The influence of the acromioclavicular joint degeneration on supraspinatus outlet impingement and the acromion shape. J Orthop Surg (Hong Kong) 2009; 17: 331-4.

2. Aydin A, Yildiz V, Kalali F, Yildirim OS, Topal M, Dostbil A. The role of acromion morphology in chronic subacromial impingement syndrome. Acta Orthop Belg 2011; 77: 733-6.

3. Balke M, Banerjee M, Vogler T, Akoto R, Bouillon B, Liem D. Acromial morphology in patients with calcific tendinitis of the shoulder. Knee Surg Sports Traumatol Arthrosc 2014; 22: 415-21.

4. Shin KM. Partial-thickness rotator cuff tears. Korean J Pain 2011; 24: 69-73.

5. Hughes PC, Green RA, Taylor NF. Measurement of subacromial impingement of the rotator cuff. J Sci Med Sport 2012; 15: 2-7.

6. Seeger LL, Gold RH, Bassett LW, Ellman H. Shoulder impingement syndrome: MR findings in 53 shoulders. AJR Am J Roentgenol 1988; 150: 343-7.

7. Balke M, Schmidt C, Dedy N, Banerjee M, Bouillon B, Liem D. Correlation of acromial morphology with impingement syn- drome and rotator cuff tears. Acta Orthop 2013; 84: 178-83.

8. Natsis K, Tsikaras P, Totlis T, Gigis I, Skandalakis P, Appell $\mathrm{HJ}$, et al. Correlation between the four types of acromion and the existence of enthesophytes: a study on 423 dried scapulas and review of the literature. Clin Anat 2007; 20: 267-72.

9. Saha S, Vasudeva N. Morphometric evaluation of adult acromion process in North Indian population. J Clin Diagn Res 2017; 11: AC08-11.

10. Kanatli U, Gemalmaz HC, Ozturk BY, Voyvoda NK, Tokgoz $\mathrm{N}$, Bolukbasi S. The role of radiological subacromial distance measurements in the subacromial impingement syndrome. Eur J Orthop Surg Traumatol 2013; 23: 317-22.

11. Li X, Xu W, Hu N, Liang X, Huang W, Jiang D, et al. Relationship between acromial morphological variation and subacromial impingement: a three-dimensional analysis. PLoS One 2017; 12: e0176193.

12. Yamaguchi K, Ditsios K, Middleton WD, Hildebolt CF, Galatz LM, Teefey SA. The demographic and morphological features of rotator cuff disease. A comparison of asymptomatic and symptomatic shoulders. J Bone Joint Surg Am 2006; 88: 1699704.

13. Edelson JG, Taitz C. Bony anatomy of coracoacromial arch: implications for arthroscopic portal placement in the shoulder. Arthroscopy 1993; 9: 201-8.

14. Zuckerman JD, Kummer FJ, Cuomo F, Simon J, Rosenblum $\mathrm{S}$, Katz N. The influence of coracoacromial arch anatomy on rotator cuff tears. J Shoulder Elbow Surg 1992; 1: 4-14.

15. Mansur DI, Khanal K, Haque MK, Sharma K. Morphometry of acromion process of human scapulae and its clinical importance amongst Nepalese population. Kathmandu Univ Med J (KUMJ) 2012; 10: 33-6.

16. Gagey N, Ravaud E, Lassau JP. Anatomy of the acromial arch: correlation of anatomy and magnetic resonance imaging. Surg Radiol Anat 1993; 15: 63-70.

17. Kitay GS, Iannotti JP, Williams GR, Haygood T, Kneeland BJ, Berlin J. Roentgenographic assessment of acromial morphologic condition in rotator cuff impingement syndrome. J Shoulder Elbow Surg 1995; 4: 441-8.

18. Banas MP, Miller RJ, Totterman S. Relationship between the lateral acromion angle and rotator cuff disease. J Shoulder Elbow Surg 1995; 4: 454-61.

19. Nyffeler RW, Werner CM, Sukthankar A, Schmid MR, Gerber C. Association of a large lateral extension of the acromion with rotator cuff tears. J Bone Joint Surg Am 2006; 88: 800-5.

20. Mayerhoefer ME, Breitenseher MJ, Wurnig C, Roposch A. Shoulder impingement: relationship of clinical symptoms and imaging criteria. Clin J Sport Med 2009; 19: 83-9.

21. Takase K, Yamamoto K. Histological and ultrastructural changes in the undersurface of the acromion with subacromial impingement. Acta Orthop 2005; 76: 386-91.

22. McGinley JC, Agrawal S, Biswal S. Rotator cuff tears: association with acromion angulation on MRI. Clin Imaging 2012; 
36: 791-6.

23. Michener LA, Subasi Yesilyaprak SS, Seitz AL, Timmons MK, Walsworth MK. Supraspinatus tendon and subacromial space parameters measured on ultrasonographic imaging in subacromial impingement syndrome. Knee Surg Sports Traumatol Arthrosc 2015; 23: 363-9. 